Research Article

\title{
Fuzzy Adaptive Variable Structure Control of Second-Order Robotic Manipulators with Dead Zones
}

\author{
Songyu Wang (iD) and Xianmin Hou (iD) \\ School of Information and Management Sciences, Henan Agricultural University, Zhengzhou 450046, China \\ Correspondence should be addressed to Songyu Wang; wangsongyu@henau.edu.cn
}

Received 27 November 2020; Revised 26 January 2021; Accepted 3 February 2021; Published 19 February 2021

Academic Editor: Shenggang Li

Copyright (c) 2021 Songyu Wang and Xianmin Hou. This is an open access article distributed under the Creative Commons Attribution License, which permits unrestricted use, distribution, and reproduction in any medium, provided the original work is properly cited.

In this study, with respect to certain second-order robotic systems with dead zones, a fuzzy adaptive variable structure controller (VSC) is implemented. Some suitable adaptive fuzzy systems are used to estimate uncertain functions. Based on Lyapunov stability theorems, parameter adaptive laws are designed, and it is proven that all signals involved will remain bounded and the stability of the controlled system is also guaranteed. Our controller is effective for the system with or without sector nonlinearity. Finally, a simulation example is presented to illustrate the correctness of the theoretical derivation.

\section{Introduction}

In the real world, almost all systems are multivariate in essence, and the multivariate control theory system can be immediately applied to various problems (aerospace technology, electric motors, and robot technology) [1-4]. Because of the coupling between control inputs and outputs, how to design an effective controller for multivariable systems is a challenging work. When multivariable systems are subject to system uncertainties, the control problems become more complicated $[5,6]$. In this field, theoretical results and constructive procedures for designing satisfactory controllers are constantly evolving, and only a few works have been reported up to now.

In recent years, fuzzy logic system (FLS) has been shown to be one of the powerful tools in functional approximately. Fuzzy control mainly utilizes the basic ideas of fuzzy mathematics and theoretical control methods. With respect to a traditional control method, the accuracy of the dynamic mode of the control system is the most important factor affecting the control performance. The more detailed the system dynamic information is, the more precise the control can be achieved [7-13]. But, with respect to a complicated system, because there are a lot of system variables, it is very hard to accurately model the system, so scholars utilize kinds of methods to describe dynamics to achieve certain purposes. That is, the traditional control theory has a strong control ability for known systems, but it is powerless for systems that are too complicated or difficult to accurately describe. So scholars try to deal with these control problems via FLS. In [9], an adaptive fuzzy control (AFC) method was proposed for a chaotic system with unknown control direction. In [11], an AFC backstepping method was proposed, where it is shown that the FLS has a very powerful approximation ability. An adaptive fuzzy observer was proposed in [13]. For more related works about the FLC, one can refer to $[4,14-19]$ and the references therein.

Since the 1980s, the control of robots has become a very meaningful work with a wide range of practical applications and has been increasingly studied by experts and scholars in various fields. The robot model is highly coupled and complex. In the early research on robot control, researchers often need to know the specific model of the robot. For some robots that are easy to model, many control methods have been proposed, such as active control, inversion based control, and backstepping control [20-23]. However, in real life, robot systems often contain many uncertainties, such as modeling estimation errors, system external disturbances, and parameter measurement errors. If the unknowns of these systems cannot be handled properly, it will affect the stability of the system and 
even make the robot system uncontrollable. Consequently, FLSs are used to tackle the uncertainties in the robotic systems. For example, in $[8,24,25]$, FLSs were used to model the robotic manipulators, and the model errors are satisfactory. More results can be referred to [24, 26-29]. However, the stability of the complicated robotic systems is hard to be theoretically discussed in the above literature.

There are two main reasons that derive the investigation of this work. First, although many control strategies have been proposed for robotic manipulators, the dead-zone input has been rarely considered. Secondly, in the traditional VSC method, the chattering phenomenon does exist, and how to solve this problem is a challenging work. Inspired by the above discussion, in this paper, an adaptive fuzzy VSC method is proposed for an $n$-link robotic manipulator with dead zone. The main contributions of this work are listed as follows: (1) to handle the dead zone in the robotic manipulator, an adaptive fuzzy VSC method is proposed, and the VSC can estimate the dead-zone model, and (2) the stability of the controlled robotic manipulator is proven strictly based on Lyapunov stability criteria.

\section{Preliminaries and Problem Description}

Consider the following $n$-link robotic manipulator:

$$
M(\xi) \ddot{\xi}+C(\xi, \dot{\xi}) \dot{\xi}+G(\xi)=\Phi(u)+a(t) .
$$

From system (1), we can get the following formula:

$$
\begin{aligned}
\ddot{\xi}= & -M^{-1}(\xi) C(\xi, \dot{\xi}) \dot{\xi}-M^{-1}(\xi) G(\xi)+M^{-1}(\xi) \Phi(u) \\
& +M^{-1}(\xi) a(t) .
\end{aligned}
$$

So, the robot system (2) is rewritten as

$$
\ddot{y}=F(x)+G(x) \Phi(u)+D(t),
$$

where

$$
\left\{\begin{array}{l}
F(x)=-M^{-1}(\xi) C(q, \dot{\xi}) \dot{\xi}-M^{-1}(\xi) G(\xi) \\
G(x)=M^{-1}(\xi) \\
D(t)=M^{-1}(\xi) a(t)
\end{array}\right.
$$

$F(),. \Phi(),. D(.) \in R^{n}, G(.) \in R^{n \times n}, y=\left[y_{1}, y_{2}, \ldots, y_{n}\right]^{T}$ $=\left[\xi_{1}, \xi_{2}, \ldots, \xi_{n}\right]^{T} \in R^{n}$ is the output, $x=\left[y_{1}, \dot{y}_{1}, \ldots\right.$, $\left.y_{n}, \dot{y}_{n}\right]^{T}=\left[\xi_{1}, \xi_{1}, \ldots, \xi_{n}, \dot{\xi}_{n}\right]^{T} \in R^{2 n}$ is the measurable state, $u=\left[u_{1}, u_{2}, \ldots, u_{n}\right]^{T} \in R^{n}$ denotes the controller to be designed, $f_{i}(x)$ is unknown, $g_{i j}, i, j=1,2, \ldots, n$ is an unknown constant control gain, $\Phi(u)=\left[\Phi_{1}\left(u_{1}\right), \Phi_{2}\left(u_{2}\right)\right.$, $\left.\ldots, \Phi_{n}\left(u_{n}\right)\right]^{T}$ means dead zone, and $d_{i}(t)$ is an external disturbance.

Let us denote

$$
\begin{aligned}
\ddot{y} & =\left[\ddot{y}_{1}, \ddot{y}_{2}, \ldots, \ddot{y}_{n}\right]^{T}, \\
F(x) & =\left[f_{1}(x), f_{2}(x), \ldots, f_{n}(x)\right]^{T}, \\
D(t) & =\left[d_{1}(t), \ldots, d_{n}(t)\right]^{T} .
\end{aligned}
$$

Now, we need the following assumptions.
Assumption 1. We assume that the desired trajectory vector $x_{d}=\left[y_{d 1}, \dot{y}_{d 1}, \ldots, y_{d n}, \dot{y}_{d n}, \ddot{y}_{d 1}, \ldots, \ddot{y}_{d n}\right]^{T}$ is continuous and bounded and can be obtained from the measurement. So $x_{d} \in \Omega_{x d} \subset R^{2 n+n}$, where $\Omega_{x d}$ is an open compact set with a finite radius.

\section{Assumption 2}

(i) Without loss of generality, one assumes that $G(x)$ is symmetric and positive definite. Therefore, there is an unknown positive constant $\delta_{g 0}$, such that $G(x) \geq \delta_{g 0} E_{n}$, where $E_{n}$ is the identity matrix.

(ii) We assume that this internal disturbance $d_{i}(t)$ is bounded and satisfies the following property: $\left|d_{i}(t)\right| \leq \bar{d}_{i}, \forall i=1,2, \ldots, n$, where $\bar{d}_{i}$ is an unknown positive constant.

Remark 1. It should be noted that, for a robotic system, Assumption 2 is reasonable, and this assumption guarantees the controllability of system (3).

The main purpose is to find a way to implement $u$ so that the output vector $y$ can better follow the desired tracking vector $y_{d}=\left[y_{d 1}, \ldots, y_{d n}\right]^{T} \in R^{n}$, and we assume that the signals involved are bounded.

Define

$$
\begin{aligned}
& e=y-y_{d}, \\
& S=\gamma e+\dot{e},
\end{aligned}
$$

where $\gamma>0$. Here, $S$ is a sliding surface variable. Then, we have

$$
\dot{S}=\gamma \dot{e}+\ddot{e}=\gamma \dot{e}+\ddot{y}-\ddot{y}_{d} .
$$

Thereafter, (7) will be used in the controller design.

2.1. Description of the Fuzzy Logic System. The basic mechanism of a fuzzy logic system includes a fuzzy inference engine and a fuzzifier, some fuzzy IF-THEN rules, and a defuzzifier.

The $i$-th fuzzy rule is in the following form: $R^{(i)}$ : if $x_{1}$ is $B_{1}^{i}$ and $\ldots$ and $x_{n}$ is $B_{n}^{i}$, then $\hat{f}$ is $f^{i}$, where $B_{1}^{i}, B_{2}^{i}, \ldots, B_{n}^{i}$ are fuzzy sets and $f^{i}$ denotes the singleton for the output in the $i$-th rule. The FLS can be rewritten as follows:

$$
\widehat{f}(\bar{x})=\frac{\sum_{i=1}^{l} f^{i}\left(\prod_{j=1}^{n} v_{B_{j}^{i}}\left(x_{j}\right)\right)}{\sum_{i=1}^{l}\left(\prod_{j=1}^{n} v_{B_{j}^{i}}\left(x_{j}\right)\right)}=\theta^{T} \varrho(\bar{x}),
$$

where $v_{B^{i}}\left(x_{j}\right)$ is the degree of membership, $\theta^{T}=\left[f^{1}, \ldots\right.$, $\left.f^{l}\right]$ can be adjusted online, and $\varrho^{T}=\left[\varrho^{1}, \ldots, \varrho^{l}\right]$, with

$$
\varrho^{i}(\bar{x})=\frac{\prod_{j=1}^{n} \nu_{B_{j}^{i}}\left(x_{j}\right)}{\sum_{i=1}^{l}\left(\prod_{j=1}^{n} v_{B_{j}^{i}}\left(x_{j}\right)\right) .}
$$

Throughout the paper, we always assume that $\sum_{i=1}^{l}\left(\prod_{j=1}^{n} v_{B_{j}^{i}}\left(x_{j}\right)\right)>0$. 
2.2. Input Nonlinearity. Suppose that $\Phi_{i}\left(u_{i}\right)$ satisfies

$$
\Phi_{i}\left(u_{i}\right)= \begin{cases}\phi_{i+}\left(u_{i}\right)\left(u_{i}-u_{i+}\right), & u_{i}>u_{i+}, \\ 0, & -u_{i-} \leq u_{i} \leq u_{i+} \\ \phi_{i-}\left(u_{i}\right)\left(u_{i}+u_{i-}\right), & u_{i}<-u_{i-},\end{cases}
$$

where $\phi_{i+}\left(u_{i}\right)>0$ and $\phi_{i-}\left(u_{i}\right)>0$ are functions with respect to $u_{i}$ and $u_{i+}>0, u_{i-}>0$.

Suppose that $\Phi_{i}\left(u_{i}\right)$ satisfies

$$
\begin{cases}\left(u_{i}-u_{i+}\right) \Phi_{i}\left(u_{i}\right) \geq l_{i+}^{*}\left(u_{i}-u_{i+}\right)^{2}, & u_{i}>u_{i+}, \\ \left(u_{i}+u_{i-}\right) \Phi_{i}\left(u_{i}\right) \geq l_{i-}^{*}\left(u_{i}+u_{i-}\right)^{2}, & u_{i}<-u_{i-},\end{cases}
$$

where $l_{i+}^{*}$ and $l_{i-}^{*}$ are constants called "gain reduction tolerances". We can write $\zeta_{i}=\min \left\{l_{i+}^{*}, l_{i-}^{*}\right\}$.

In order to facilitate the study of the characteristics of this input nonlinear function in the control problems, we give the following assumption.

Assumption 3. Suppose that $l_{i+}^{*}$ and $l_{i-}^{*}$ are unknown, that is, $\zeta_{i}$ is also unknown. The explicit mathematical expression of the nonlinear function $\Phi_{i}\left(u_{i}\right)$ is uncertain, but this property (11) and constants $u_{i+}$ and $u_{i-}$ are known.

Remark 2. One knows from (10) and (11) that, for $u_{i+}=u_{i-}=0$, the input $\Phi_{i}\left(u_{i}\right)$ is reduced to the special sector nonlinear function. We can make the following restriction: the gain reduction tolerances $l_{i+}^{*}$ and $l_{i-}^{*}$ and their $\operatorname{minimum} \zeta_{i}=\min \left\{l_{i+}^{*}, l_{i-}^{*}\right\}$ are known.

\section{Main Results}

In this article, a fuzzy adaptive VSC scheme is proposed for a class of two-link robotic manipulator systems described in (3).

By bringing (3) into (7), the dynamic equation of $S$ can become

$$
\dot{S}=\gamma \dot{e}+F(x)+G(x) \Phi(u)+D(t)-\ddot{y}_{d} .
$$
have

Posing $G_{1}(x)=G^{-1}(x)$ and $D_{h}(t)=G^{-1}(x) D(t)$, we

$$
\begin{aligned}
G^{-1}(x) \dot{S}= & \gamma G^{-1}(x) \dot{e}+G^{-1}(x) F(x)+\Phi(u) \\
& +G^{-1}(x) D(t)-G^{-1}(x) \ddot{y}_{d},
\end{aligned}
$$

Then, $S$ can be expressed by

$$
\begin{aligned}
G_{1}(x) \dot{S} & =G_{1}(x)\left[\gamma \dot{e}+F(x)-\ddot{y}_{d}\right]+\Phi(u)+D_{h}(t) \\
& =\beta(x, \omega)+\Phi(u)+D_{h}(t),
\end{aligned}
$$

where $\beta(x, \omega)=\left[\beta_{1}(x, \omega), \ldots, \beta_{n}(x, \omega)\right]^{T}=G_{1}(x)[\omega+F(x)]$ and $\omega=\gamma \dot{e}-\ddot{y}_{d}$.

Assumption 4. One can find a function $\bar{\beta}_{i}(x)$ such that

$$
\left|\beta_{i}(x, \omega)\right| \leq \zeta \bar{\beta}_{i}(x), \quad \forall x \in \Omega_{x} \subset R^{2 n},
$$

where $\zeta=\min _{i}\left\{\zeta_{i}\right\}$.
Remark 3. Assumption 4 is reasonable because

(i) $\zeta=\min _{i}\left\{\zeta_{i}\right\} a \zeta \bar{\beta}_{i}(x)$ is unknown, where is $\zeta$ is a positive scalar

(ii) Nothing that $\omega$ is a function of $\left(x, x_{d}\right), x_{d} \in L_{\infty}$, and $\beta_{i}(x, \omega)$ is a continuous function; therefore, such a function $\bar{\beta}_{i}(x)$ always exists

Multiplying (14) by $S^{T}$ and using Assumption 4, we have

$$
\begin{aligned}
\frac{1}{\zeta} S^{T} G_{1}(x) \dot{S} & =\frac{1}{\zeta} S^{T} \beta(x, \omega)+\frac{1}{\zeta} S^{T} \Phi(u)+\frac{1}{\zeta} S^{T} D_{h}(t) \\
& =\frac{1}{\zeta} \sum_{i=1}^{n} S_{i} \beta_{i}(x, \omega)+\frac{1}{\zeta} S^{T} \Phi(u)+\frac{1}{\zeta} S^{T} D_{h}(t) \\
& \leq \frac{1}{\zeta} \sum_{i=1}^{n}\left|S_{i}\right|\left|\beta_{i}(x, \omega)\right|+\frac{1}{\zeta} S^{T} \Phi(u)+\frac{1}{\zeta} S^{T} D_{h}(t) \\
& \leq \sum_{i=1}^{n}\left|S_{i}\right| \bar{\beta}_{i}(x)+\frac{1}{\zeta} S^{T} \Phi(u)+\frac{1}{\zeta} S^{T} D_{h}(t) .
\end{aligned}
$$

On a compact set $\Omega_{x}$, the unknown continuous nonlinear function $\bar{\beta}_{i}(x)$ can be approximated by the fuzzy system (8) as follows:

$$
\widehat{\overline{\beta_{i}}}\left(x, \theta_{i}\right)=\theta_{i}^{T} \varrho_{i}(x) .
$$

Next, we can define the optimal parameter as

$$
\theta_{i}^{*}=\arg \min _{\theta_{i}}\left[\sup _{x \in \Omega_{x}}\left|\overline{\beta_{i}}(x)-\widehat{\bar{\beta}}_{i}\left(x, \theta_{i}\right)\right|\right] .
$$

Define

$$
\tilde{\theta}_{i}=\theta_{i}-\theta_{i}^{*}
$$

as the parameter estimation error, with

$$
\varepsilon_{i}(x)=\overline{\beta_{i}}(x)-\hat{\bar{\beta}}_{i}\left(x, \theta_{i}^{*}\right),
$$

being the approximation error, with $\widehat{\bar{\beta}_{i}}\left(x, \theta_{i}^{*}\right)=\theta_{i}^{T *} \varrho_{i}(x)$.

As in the literature, the fuzzy systems used on the compact set $\Omega_{x}$ do not violate this universal approximation property. So, we can assume that

$$
\left|\varepsilon_{i}(x)\right| \leq \bar{\varepsilon}_{i}, \quad \forall x \in \Omega_{x},
$$

with $\overline{\varepsilon_{i}}$ being unknown.

Then, one has

$$
\begin{aligned}
\widehat{\bar{\beta}_{i}}\left(x, \theta_{i}\right)-\overline{\beta_{i}}(x) & =\overline{\bar{\beta}}_{i}\left(x, \theta_{i}\right)-\widehat{\overline{\beta_{i}}}\left(x, \theta_{i}^{*}\right)+\overline{\bar{\beta}}_{i}\left(x, \theta_{i}^{*}\right)-\overline{\beta_{i}}(x) \\
& =\overline{\bar{\beta}}_{i}\left(x, \theta_{i}\right)-\overline{\bar{\beta}_{i}}\left(x, \theta_{i}^{*}\right)-\varepsilon_{i}(x) \\
& =\theta_{i}^{T} \varrho_{i}(x)-\theta_{i}^{* T} \varrho_{i}(x)-\varepsilon_{i}(x) \\
& =\widetilde{\theta}_{i}^{T} \varrho_{i}(x)-\varepsilon_{i}(x) .
\end{aligned}
$$

From (16) and (22), we can get 


$$
\begin{aligned}
\frac{1}{\zeta} S^{T} G_{1}(x) \dot{S} \leq & \sum_{i=1}^{n}\left|S_{i}\right| \bar{\beta}_{i}(x)+\frac{1}{\zeta} S^{T} \Phi(u)+\frac{1}{\zeta} S^{T} D_{h}(t) \\
= & \sum_{i=1}^{n}\left|S_{i}\right|\left(\theta_{i}^{T} \varrho_{i}(x)-\tilde{\theta}_{i}^{T} \varrho_{i}(x)+\varepsilon_{i}(x)\right)+\frac{1}{\zeta} S^{T} \Phi(u) \\
& +\frac{1}{\zeta} S^{T} D_{h}(t) \\
\leq & \sum_{i=1}^{n}\left|S_{i}\right| \theta_{i}^{T} \varrho_{i}(x)-\sum_{i=1}^{n}\left|S_{i}\right| \tilde{\theta}_{i}^{T} \varrho_{i}(x)+\sum_{i=1}^{n}\left|S_{i}\right|\left|\varepsilon_{i}(x)\right| \\
& +\frac{1}{\zeta} S^{T} \Phi(u)+\left|\frac{1}{\zeta} S^{T} D_{h}(t)\right| \\
\leq & \sum_{i=1}^{n}\left|S_{i}\right| \theta_{i}^{T} \varrho_{i}(x)-\sum_{i=1}^{n}\left|S_{i}\right| \widetilde{\theta}_{i}^{T} \varrho_{i}(x)+\sum_{i=1}^{n}\left|S_{i}\right| \bar{\varepsilon}_{i} \\
& +\sum_{i=1}^{n}\left|S_{i}\right| \widetilde{\kappa_{0 i}}+\sum_{i=1}^{n}\left|S_{i}\right| \kappa_{0 i}, \\
\leq & \sum_{i=1}^{n}\left|S_{i}\right| \theta_{i}^{T} \varrho_{i}(x)-\sum_{i=1}^{n}\left|S_{i}\right| \widetilde{\theta}_{i}^{T} \varrho_{i}(x)+\frac{1}{\zeta} S^{T} \Phi(u)+\sum_{i=1}^{n}\left|S_{i}\right| \bar{d}_{h i} \\
& 1(x) \\
&
\end{aligned}
$$

where $\widetilde{\theta}_{i}=\theta_{i}-\theta_{i}^{*}$ and $\widetilde{\kappa_{0 i}}=\kappa_{0 i}-\kappa_{0 i}^{*}=\kappa_{0 i}-\overline{\varepsilon_{i}}-\overline{d_{h i}}$. Note that $\overline{d_{h i}}$ are unknown positive constants which satisfy $\sum_{i=1}^{n} \overline{d_{h i}}\left|S_{i}\right| \geq\left|S^{T} D_{h}(t) / \zeta\right|$.

In order to meet the control goal, we propose a suitable fuzzy adaptive VSC as follows:

$$
u_{i}= \begin{cases}-\omega_{i}(t) \operatorname{sign}\left(S_{i}\right)-u_{i-}, & S_{i}>0, \\ 0, & S_{i}=0, \\ -ळ_{i}(t) \operatorname{sign}\left(S_{i}\right)+u_{i+}, & S_{i}<0,\end{cases}
$$

with

$$
\begin{aligned}
ळ_{i}(t) & =\kappa_{0 i}+\kappa_{1 i}\left|S_{i}\right|+\theta_{i}^{T} \varrho_{i}(x), \quad \forall i=1,2, \ldots, n, \\
\dot{\kappa}_{0 i} & =-\chi_{0 i} \delta_{0 i} k_{0 i}+\chi_{0 i}\left|S_{i}\right|, \quad \kappa_{0 i}(0) \geq 0, \\
\dot{\theta}_{i} & =-\chi_{1 i} \delta_{1 i} \theta_{i}+\chi_{1 i}\left|S_{i}\right| \varrho_{i}(x), \quad \theta_{i j}(0) \geq 0,
\end{aligned}
$$

where $\chi_{0 i}, \chi_{1 i}, \delta_{0 i}, \delta_{1 i}, \kappa_{1 i}>0$, and $\kappa_{0 i}$ and $\theta_{i}$ are the online estimates of the uncertain terms $\kappa_{0 i}^{*}=\overline{\varepsilon_{i}}+\overline{d_{h i}}$ and $\theta_{i}^{*}$, respectively. $S_{i}>0$,

From (11) and (24), we can easily get that $u_{i}<-u_{i-}$ for

$$
\begin{aligned}
& \left(u_{i}+u_{i-}\right) \Phi_{i}\left(u_{i}\right)=-\Phi_{i}(t) \operatorname{sign}\left(S_{i}\right) \Phi_{i}\left(u_{i}\right) \geq l_{i-}^{*}\left(u_{i}+u_{i-}\right)^{2} \\
& =l_{i-}^{*} \omega_{i}^{2}(t) \geq \zeta \omega_{i}^{2}(t),
\end{aligned}
$$

and $u_{i}>u_{i+}$ for $S_{i}<0$,

$$
\begin{aligned}
\left(u_{i}-u_{i+}\right) \Phi_{i}\left(u_{i}\right) & =-ळ_{i}(t) \operatorname{sign}\left(S_{i}\right) \Phi_{i}\left(u_{i}\right) \geq l_{i+}^{*}\left(u_{i}-u_{i+}\right)^{2} \\
& =l_{i+}^{*} \Phi_{i}^{2}(t) \geq \zeta \Phi_{i}^{2}(t) .
\end{aligned}
$$

Then, for $S_{i}>0$ and for $S_{i}<0$, we have

$$
-\varpi_{i}(t) \operatorname{sign}\left(S_{i}\right) \Phi_{i}\left(u_{i}\right) \geq \zeta ळ_{i}^{2}(t)
$$

Next, we can use $S_{i}^{2} \geq 0$ and $S_{i} \operatorname{sign}\left(S_{i}\right)=\left|S_{i}\right|$, and we get

$$
-\varpi_{i}(t) S_{i}^{2} \operatorname{sign}\left(S_{i}\right) \Phi_{i}\left(u_{i}\right) \geq \zeta \varpi_{i}^{2}(t) S_{i}^{2}=\zeta \varpi_{i}^{2}(t)\left|S_{i}\right|^{2} .
$$

Finally, for all $S_{i}$ (i.e., for $S_{i}>0, S_{i}=0$, and $S_{i}<0$ ), we get

$$
S_{i} \Phi_{i}\left(u_{i}\right) \leq-\zeta \varpi_{i}(t)\left|S_{i}\right|
$$

Theorem 1. For the robotic manipulator (3) with Assumptions 1-4, the proposed adaptive fuzzy VSC (24)-(27) guarantee that the tracking error converges to an adjustable region.

Proof. Let

$$
V=\frac{1}{2 \zeta} S^{T} G_{1}(x) S+\frac{1}{2} \sum_{i=1}^{n} \frac{1}{\chi_{0 i}} \widetilde{\kappa_{0 i}^{2}}+\frac{1}{2} \sum_{i=1}^{n} \frac{1}{\chi_{1 i}} \widetilde{\theta_{i}^{T}} \widetilde{\theta}_{i} .
$$

Its time derivative is given by

$$
\dot{V}=\frac{1}{2 \zeta} S^{T} G_{1}(x) S+\sum_{i=1}^{n} \frac{1}{\chi_{0 i}} \widetilde{\kappa_{0 i}} \dot{\kappa}_{0 i}+\sum_{i=1}^{n} \frac{1}{\chi_{1 i}} \widetilde{\theta_{i}^{T}} \dot{\theta}_{i},
$$

with $\dot{G}_{1}(x)=0$.

Using expressions (16), (26), (27), and (32), (34) can become

$$
\begin{aligned}
\dot{V} \leq & \sum_{i=1}^{n}\left|S_{i}\right| \kappa_{0 i}+\sum_{i=1}^{n}\left|S_{i}\right| \theta_{i}^{T} \varrho_{i}(x)+\frac{1}{\zeta} \sum_{i=1}^{n} S_{i} \Phi_{i}\left(u_{i}\right) \\
& -\sum_{i=1}^{n} \delta_{0 i} \widetilde{\kappa_{0 i}} \kappa_{0 i}-\sum_{i=1}^{n} \delta_{1 i} \widetilde{\theta_{i}^{T}} \theta_{i} \\
\leq & \sum_{i=1}^{n}\left|S_{i}\right| \kappa_{0 i}+\sum_{i=1}^{n}\left|S_{i}\right| \theta_{i}^{T} \varrho_{i}(x)+\sum_{i=1}^{n}-{\widetilde{\omega_{i}}}_{i}(t)\left|S_{i}\right| \\
& -\sum_{i=1}^{n} \delta_{0 i} \widetilde{\kappa_{0 i}} \kappa_{0 i}-\sum_{i=1}^{n} \delta_{1 i} \tilde{\theta_{i}^{T}} \theta_{i} \\
= & -\sum_{i=1}^{n} \kappa_{1 i} S_{i}^{2}-\sum_{i=1}^{n} \delta_{0 i} \widetilde{\kappa_{0 i}} \kappa_{0 i}-\sum_{i=1}^{n} \delta_{1 i} \widetilde{\theta_{i}^{T}} \theta_{i} .
\end{aligned}
$$

We can easily verify that 


$$
\begin{aligned}
& -\delta_{0 i} \widetilde{\kappa_{0 i}} \kappa_{0 i} \leq-\frac{\delta_{0 i}}{2} \widetilde{\kappa_{0 i}^{2}}+\frac{\delta_{0 i}}{2} \kappa_{0 i}^{* 2}, \\
& -\delta_{1 i} \widetilde{\theta_{i}^{T}} \theta_{i} \leq-\frac{\delta_{1 i}}{2}\left\|\widetilde{\theta}_{i}\right\|^{2}+\frac{\delta_{1 i}}{2}\left\|\theta_{i}^{*}\right\|^{2} .
\end{aligned}
$$

Using the previous inequalities, (35) becomes

$$
\dot{V} \leq-\sum_{i=1}^{n} \kappa_{1 i} S_{i}^{2}-\sum_{i=1}^{n} \frac{\delta_{0 i}}{2} \widetilde{\kappa}_{0 i}^{2}+\sum_{i=1}^{n} \frac{\delta_{0 i}}{2} \kappa_{0 i}^{* 2}-\sum_{i=1}^{n} \frac{\delta_{1 i}}{2}\left\|\widetilde{\theta}_{i}\right\|^{2}+\sum_{i=1}^{n} \frac{\delta_{1 i}}{2}\left\|\theta_{i}^{*}\right\|^{2} .
$$

Since $G(x) \geq \delta_{g 0} E_{n}$ (recall that the matrix $G(x)$ is positive definite and symmetric), then

$$
S^{T} G^{-1}(x) S=S^{T} G_{1}(x) S \leq \frac{1}{\delta_{g 0}}\|S\|^{2} .
$$

From (37) and (38), we have

$$
\dot{V} \leq-\omega V+\tau,
$$

where

$$
\begin{aligned}
\tau & =\sum_{i=1}^{n} \frac{\delta_{0 i}}{2} \kappa_{0 i}^{* 2}+\sum_{i=1}^{n} \frac{\delta_{1 i}}{2}\left\|\theta_{i}^{*}\right\|^{2}, \\
\omega & =\min \left\{\min _{i}\left\{2 \zeta \delta_{g 0} \kappa_{1 i}\right\}, \min _{i}\left\{\chi_{0 i} \delta_{0 i}\right\}, \min _{i}\left\{\chi_{1 i} \delta_{1 i}\right\}\right\} .
\end{aligned}
$$

Multiplying (39) by $e^{\omega t}$ yields

$$
\frac{\mathrm{d}}{\mathrm{d} t}\left(V e^{\omega t}\right) \leq \tau e^{\omega t}
$$

Integrating $(41)$ over $[0, t]$, we have

$$
0 \leq V(t) \leq \frac{\pi}{\omega}+\left(V(0)-\frac{\tau}{\omega}\right) e^{-\omega t} .
$$

So, all signals $\kappa_{0 i}, \theta_{i}, S_{i}$, and $x$ are bounded.

From (33), $V(0)$ can be written as follows:

$$
\begin{aligned}
V(0)= & \frac{1}{2 \zeta} S(0)^{T} G_{1}(x) S(0)+\frac{1}{2} \sum_{i=1}^{n} \frac{1}{\chi_{0 i}}\left(\kappa_{0 i}(0)-\kappa_{0 i}^{*}\right)^{2} \\
& +\frac{1}{2} \sum_{i=1}^{n} \frac{1}{\chi_{1 i}}\left(\theta_{i}(0)-\theta_{i}^{*}\right)^{T}\left(\theta_{i}(0)-\theta_{i}^{*}\right) .
\end{aligned}
$$

Since $G_{1}(x)$ is symmetric and positive definite (i.e., it exists an unknown positive constant $\delta_{g 1}$, such that $\left.G_{1}(x) \geq \delta_{g 1} E_{n}\right)$, from (33) and (42), we have

$$
\left|S_{i}\right| \leq\left(\frac{2 \zeta}{\delta_{g 1}}\left(\frac{\tau}{\omega}+\left(V(0)-\frac{\tau}{\omega}\right) e^{-\omega t}\right)\right)^{1 / 2}
$$

Finally, the solution of $S_{i}$ exponentially converges to a bounded region $\Omega_{S_{i}}=\left\{S_{i}|| S_{i} \mid \leq\left(\left(2 \zeta / \delta_{g 1}\right)(\tau / \omega)\right)^{1 / 2}\right\}$.

Remark 4. If $\Phi_{i}\left(u_{i}\right)=u_{i}$ (or when $u_{i+}=u_{i-}=0$, and $\left.\phi_{i+}\left(u_{i}\right)=\phi_{i-}\left(u_{i}\right)=1\right)$, i.e., there are no dead-zone nonlinear function terms in the input function, we can clearly show that the proposed controller is still suitable for such two-link robotic manipulator systems.

Remark 5. In the case where $u_{i+}=u_{i-}=u_{i 0},(24)$ is reduced to the following expression:

$$
u_{i}=-\left(\oplus_{i t}+u_{i 0}\right) \operatorname{sign}\left(S_{i}\right)
$$

where $\omega_{i}(t)=\kappa_{0 i}+\kappa_{1 i}\left|S_{i}\right|+\theta_{i}^{T} \varrho_{i}(x)$.

\section{Simulation Results}

In this part, a simulation example is given to show the effectiveness of the proposed adaptive fuzzy controller. System (3) is given by

$$
\ddot{\xi}=M^{-1}(\xi)[-C(\xi, \dot{\xi}) \dot{\xi}-G(\xi)+\Phi(u)]+D(t) .
$$

And the details of system model (46) are given as follows:

$$
\begin{aligned}
M(\xi) & =\left[\begin{array}{cc}
M_{11} & M_{12} \\
M_{12} & M_{22}
\end{array}\right], \\
C(\xi, \dot{\xi}) \dot{\xi} & =\left[\begin{array}{c}
-\frac{1}{2} m_{2} l_{1} l_{2}\left(2 \dot{\xi}_{1} \dot{\xi}_{2}+\dot{\xi}_{2}^{2}\right) \sin q_{2} \\
\frac{1}{2} m_{2} l_{1} l_{2} \dot{\xi}_{1}^{2} \sin \xi_{2}
\end{array}\right],
\end{aligned}
$$

$$
\begin{aligned}
& G(\xi)=\left[\begin{array}{c}
\left(\frac{1}{2} m_{1}+m_{2}\right) g l_{1} \cos \xi_{2}+\frac{1}{2} m_{2} l_{2} g \cos \left(\xi_{1}+\xi_{2}\right) \\
\frac{1}{2} m_{2} g l_{2} \cos \left(\xi_{1}+\xi_{2}\right)
\end{array}\right], \\
& D(t)=\left[\begin{array}{l}
\sin t \\
\sin t
\end{array}\right] .
\end{aligned}
$$

The input nonlinearities $\Phi_{1}\left(u_{1}\right)$ are described by

$$
\Phi_{1}\left(u_{1}\right)= \begin{cases}\left(u_{1}-2\right)\left(1-0.3 \sin \left(u_{1}\right)\right), & u_{1}>2, \\ 0, & -2 \leq u_{1} \leq 2, \\ \left(u_{1}+2\right)\left(0.8-0.3 \cos \left(u_{1}\right)\right), & u_{1}<-2,\end{cases}
$$

and the input nonlinearity $\Phi_{2}\left(u_{2}\right)$ is supposed to be

$$
\Phi_{2}\left(u_{2}\right)= \begin{cases}\left(u_{2}-5\right)\left(1-0.3 \sin \left(u_{2}\right)\right), & u_{2}>5, \\ 0, & -5 \leq u_{2} \leq 5, \\ \left(u_{2}+5\right)\left(0.8-0.3 \cos \left(u_{2}\right)\right), & u_{2}<-5 .\end{cases}
$$

Then, we have $M_{11}=\left((1 / 4) m_{1}+m_{2}\right) l_{1}^{2}+(1 / 4) m_{2} l_{2}^{2}+$ $m_{2} l_{1} l_{2} \cos q_{2}, M_{12}=(1 / 4) m_{2} l_{2}^{2}+(1 / 2) m_{2} l_{1} l_{2} \cos q_{2}$, and $M_{22}=(1 / 4) m_{2} l_{2}^{2}, m_{1}$ and $m_{2}$ are the mass, and $l_{1}$ and $l_{2}$ denote the length. In the simulation, $m_{1}=4, m_{2}=2, l_{1}=2$, $l_{2}=1$, and $g=9.8$. 

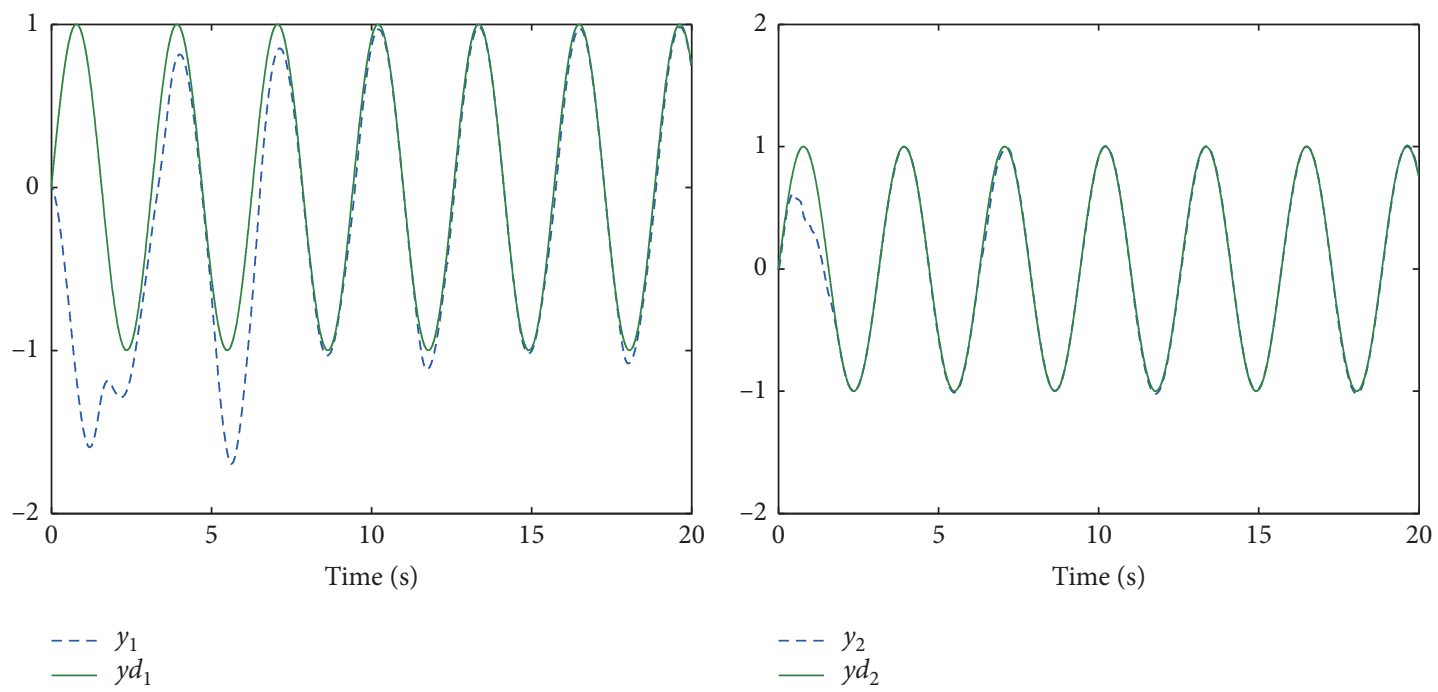

(a)

(b)

Figure 1: Simulation results for (a) $y_{1}(t)$ and (b) $y_{2}(t)$.
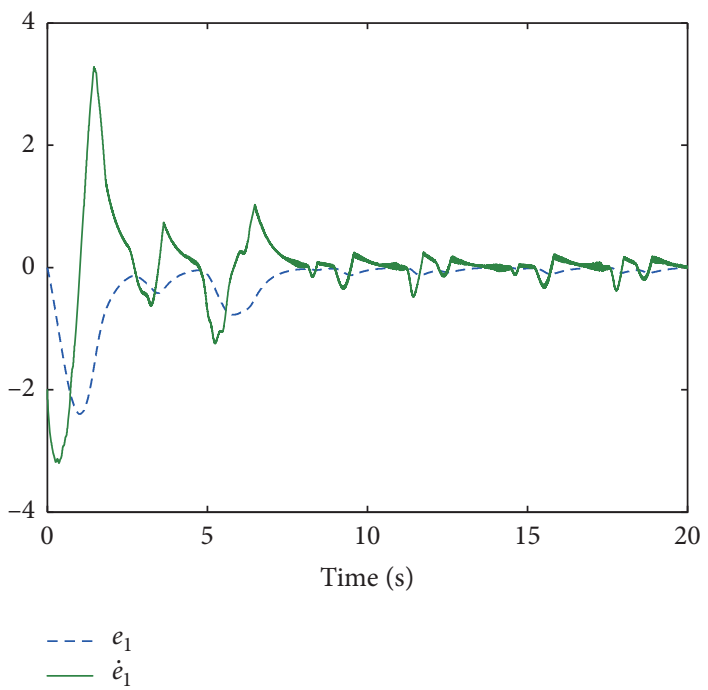

(a)

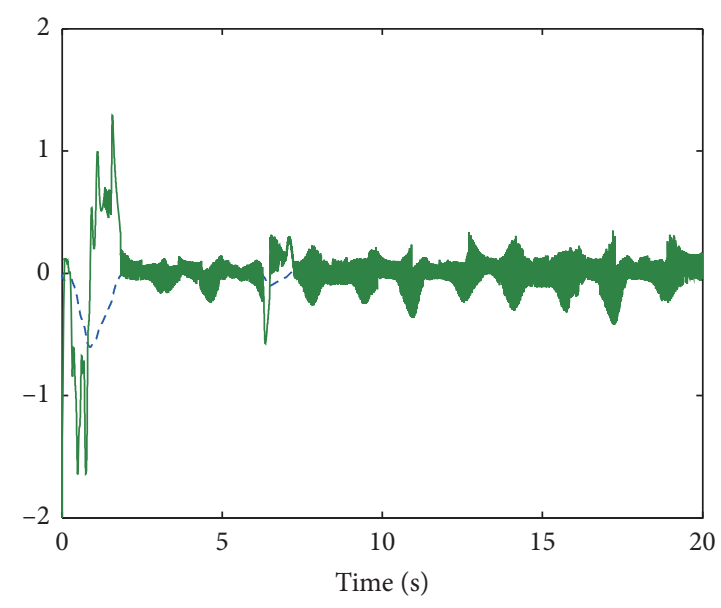

$\begin{array}{rr}--- & e_{2} \\ - & \dot{e}_{2}\end{array}$

(b)

Figure 2: Tracking errors for (a) $e_{1}$ and (b) $e_{2}$. 


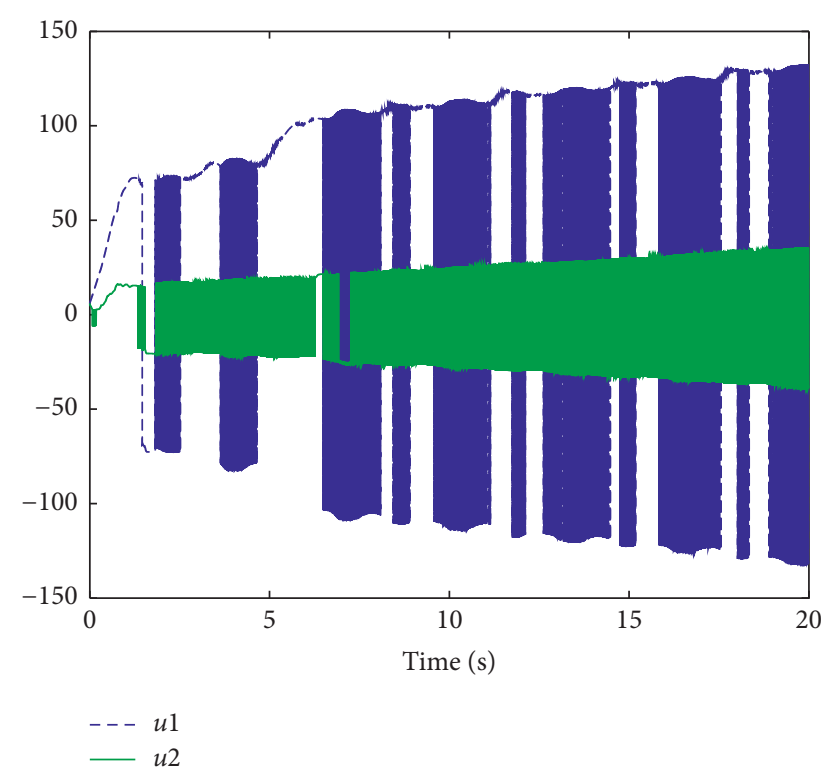

Figure 3: Control input signal for $u_{1}$ and $u_{2}$.

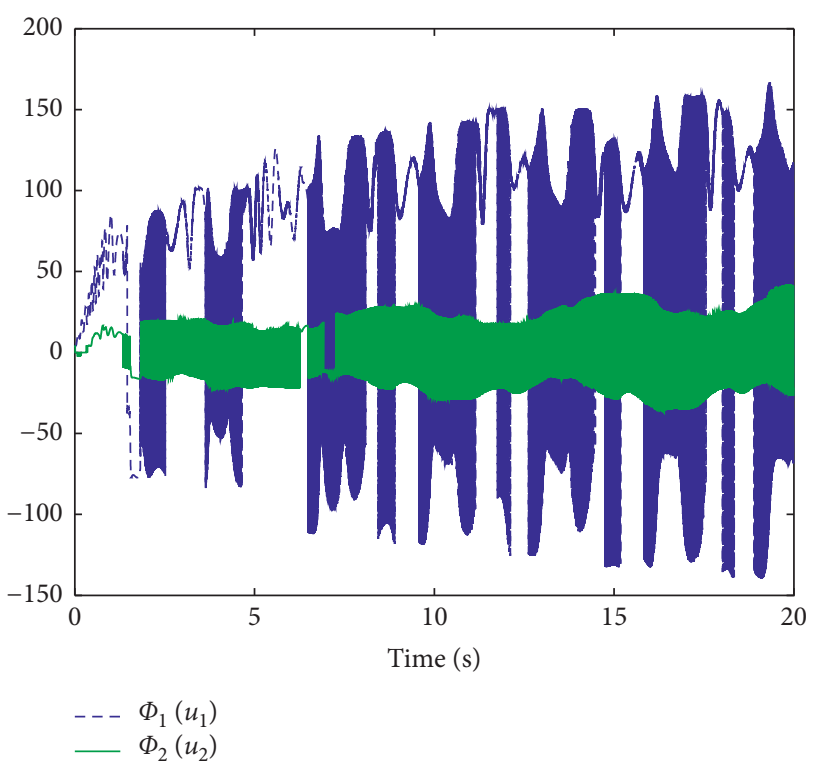

Figure 4: Control input function for $\Phi_{1}\left(u_{1}\right)$ and $\Phi_{2}\left(u_{2}\right)$.

Define $y=\left[y_{1}, y_{2}\right]^{T}=\left[\xi_{1}, \xi_{2}\right]^{T}, u=\left[u_{1}, u_{2}\right]^{T}, \Phi(u)=$ $\left[\Phi_{1}\left(u_{1}\right), \Phi_{2}\left(u_{2}\right)\right]^{T}, \quad D(t)=\left[d_{1}(t), d_{2}(t)\right]^{T}$, and $x=\left[x_{1}^{T}\right.$, $\left.x_{2}^{T}\right]^{T} \in \mathscr{R}^{4}$, where $x_{1}(t)=\left[\xi_{1}, \xi_{2}\right]^{T}$ and $x_{2}(t)=\left[\dot{\xi}_{1}, \dot{\xi}_{2}\right]^{T}$. The initial conditions of the system are taken as $x(0)=\left[y_{1}(0), y_{2}(0), \dot{y}_{1}(0), \dot{y}_{2}(0)\right]^{T}=[0,0,0,0]^{T}$. The desired signal is $y_{d}=[\sin 2 t \text {, sin } 2 t]^{T}$, and the external disturbances are selected as follows: $d_{1}(t)=d_{2}(t)=\sin t$.

The adaptive fuzzy systems $\theta_{i}^{T} \varrho_{i}(x)$, with $i=1,2$, have the vector $x=\left[y_{1}, y_{2}, \dot{y}_{1}, \dot{y}_{2}\right]^{T}$ as input, and we define three triangular membership functions uniformly distributed on the intervals $[-5,5]$, so we will use $3 \times 3 \times 3 \times 3=81$ fuzzy rules in the simulation. The design parameters are chosen as follows: $\chi_{01}=\chi_{02}=10, \chi_{11}=\chi_{12}=5, \quad \delta_{01}=\delta_{02}=0.001$, $\delta_{11}=\delta_{12}=0.0005, \gamma_{1}=\gamma_{2}=2$, and $\kappa_{11}=\kappa_{12}=2$. The

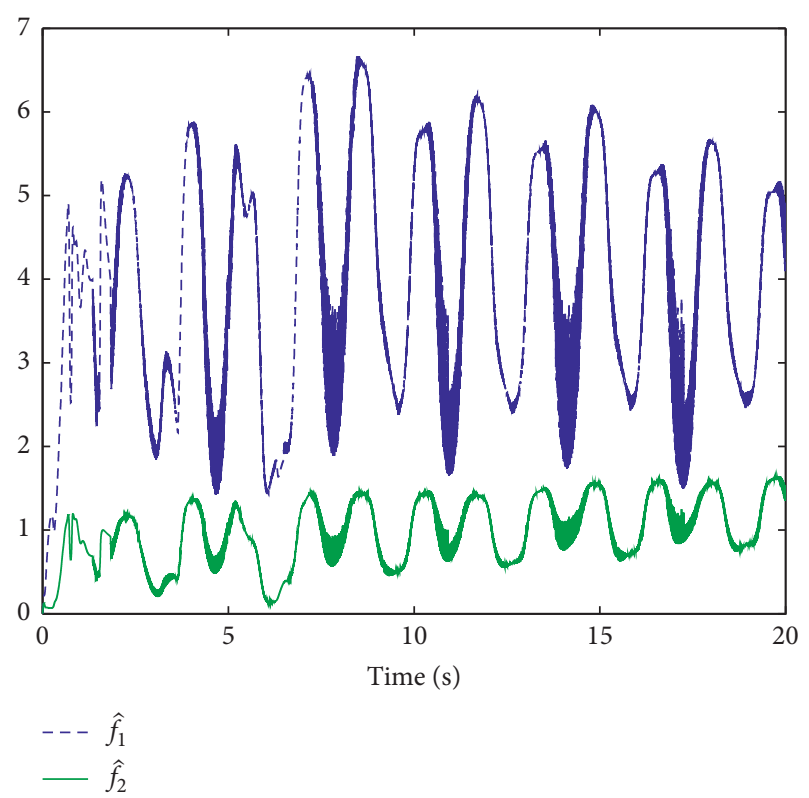

FIgURe 5: Estimation of input function for $f_{1}(x)$ and $f_{2}(x)$.

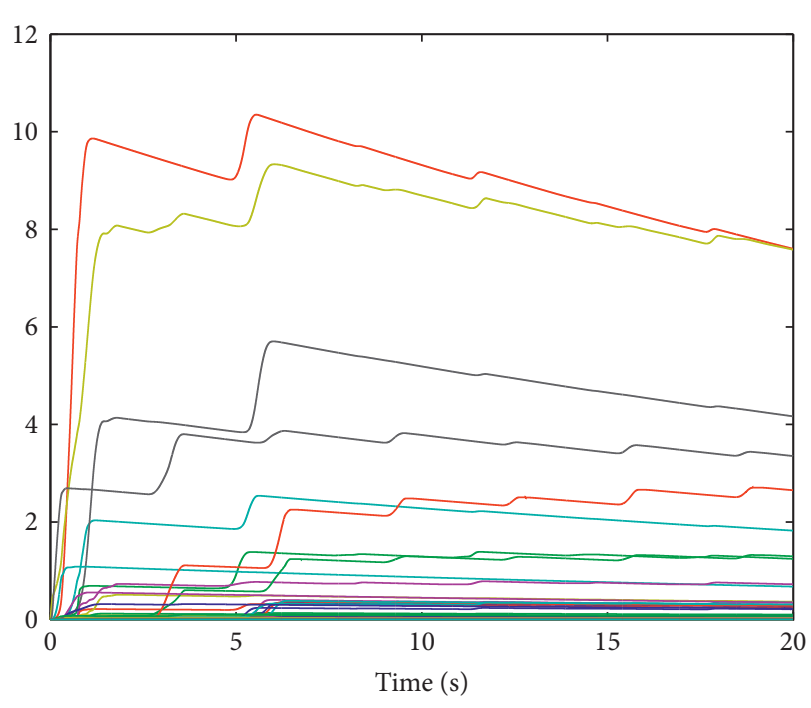

Figure 6: Fuzzy parameter for $\theta_{1}$.

initial condition is $\kappa_{01}(0)=\kappa_{02} \quad(0)=0, \theta_{1 j}=\theta_{2 j}=0$, $j=1,2, \ldots, 81$.

The simulation results are shown in Figures 1-7. Figure 1 clearly shows the simulation results of the output vector $y$ tracking the expected vector $y_{d}$, which shows that the tracking performance is very good. Figures 2 (a) and 2(b) show the tracking errors and their derivatives of the two-link robotic manipulator system, i.e., $e_{i}$ and $\dot{e}_{i}$, for $i=1,2$. Figure 3 presents the control input vector $u_{1}$ and $u_{2}$, and the boundary of the control input signal is bounded. It is worth noting that, in all these simulation results, the assumptions of the two-link robotic manipulator system model are completely unknown, but for some functions, i.e., $\Phi_{i}\left(u_{i}\right), i=1,2$ and $G(x)$, their basic properties are already known. Figure 4 shows the control input function of the system. Figure 5 shows the estimated value of the control 


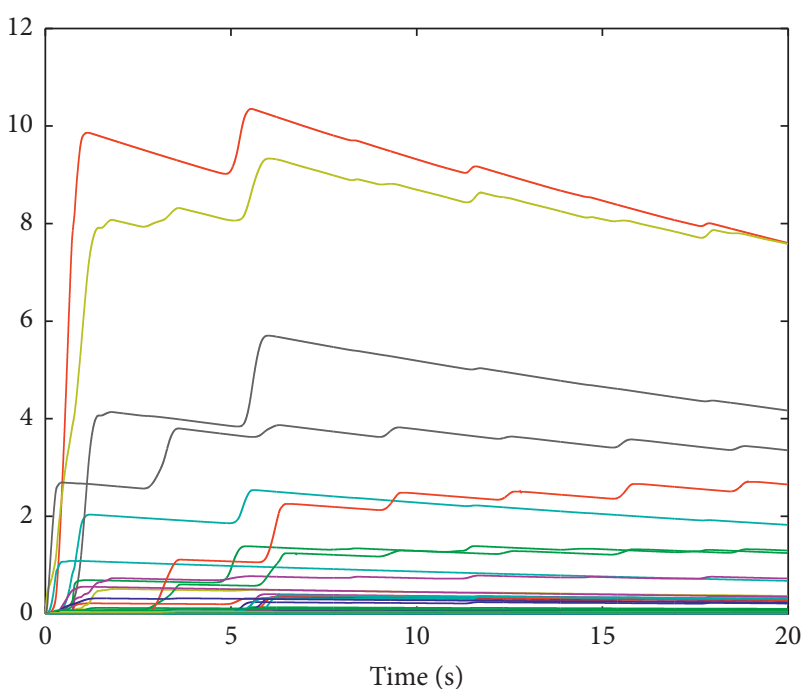

FIgURE 7: Fuzzy parameter for $\theta_{2}$.

input function. The adaptive fuzzy parameters of the fuzzy system are shown in Figures 6 and 7. Through these simulation results, we can see the boundedness of these control signals in closed-loop systems, and through the proposed fuzzy adaptive control scheme, internal disturbances in twolink robotic manipulator system and uncertain nonlinear input functions can be effectively resolved. Finally, we can get that, through the control signal, these input vectors can quickly converge to the tracking vector at time $t=0$.

\section{Conclusions}

In this study, a fuzzy adaptive VSC for uncertain robotic manipulators system with dead zones has been proposed. It is proven that the proposed VSC can well handle the dead zone. The FLSs are used to model the robotic manipulators, and it is also shown that the model error is small enough by using the proposed adaptation law. Meanwhile, the VSC can maintain the good tracking performance even in the presence of dead zones. How to extend the proposed method to tackle the input saturation is one of our research directions.

\section{Data Availability}

All data used to support the findings of this are included in the manuscript.

\section{Conflicts of Interest}

The authors declare that they have no conflicts of interest.

\section{References}

[1] K.-M. Koo, "Stable adaptive fuzzy controller with timevarying dead-zone," Fuzzy Sets and Systems, vol. 121, no. 1, pp. 161-168, 2001.

[2] S. Yu, X. Yu, B. Shirinzadeh, and Z. Man, "Continuous finitetime control for robotic manipulators with terminal sliding modes," Automatica, vol. 41, no. 11, pp. 1957-1964, 2005.
[3] A. Boulkroune and M. M'Saad, "A fuzzy adaptive variablestructure control scheme for uncertain chaotic mimo systems with sector nonlinearities and dead-zones," Expert Systems with Applications, vol. 38, no. 12, pp. 14744-14750, 2011.

[4] H. Liu, Y. Pan, and J. Cao, "Composite learning adaptive dynamic surface control of fractional-order nonlinear systems," IEEE Transactions on Cybernetics, vol. 50, no. 6, pp. 2557-2567, 2020.

[5] P. Wang, G. Wen, X. Yu, W. Yu, and Y. Lv, "Consensus disturbance rejection for linear multiagent systems with directed switching communication topologies," IEEE Transactions on Control of Network Systems, vol. 7, no. 1, pp. 254-265, 2019.

[6] P. Wang, G. Wen, T. Huang, W. Yu, and Y. Ren, "Observerbased consensus protocol for directed switching networks with a leader of nonzero inputs," IEEE Transactions on Cybernetics, pp. 1-11, 2020.

[7] T.-C. Lin and C.-H. Kuo, "Synchronization of uncertain fractional order chaotic systems: adaptive fuzzy approach," ISA Transactions, vol. 50, no. 4, pp. 548-556, 2011.

[8] N. Mendes and P. Neto, "Indirect adaptive fuzzy control for industrial robots: a solution for contact applications," Expert Systems with Applications, vol. 42, no. 22, pp. 8929-8935, 2015.

[9] H. Liu, S. G. Li, Y. G. Sun, and H. X. Wang, “Adaptive fuzzy synchronization for uncertain fractional-order chaotic systems with unknown non-symmetrical control gain," Acta Physica Sinaca, vol. 64, no. 7, Article ID 070503, 2015.

[10] R. Shahnazi, "Observer-based adaptive interval type-2 fuzzy control of uncertain mimo nonlinear systems with unknown asymmetric saturation actuators," Neurocomputing, vol. 171, pp. 1053-1065, 2016.

[11] H. Liu, Y. Pan, S. Li, and Y. Chen, "Adaptive fuzzy backstepping control of fractional-order nonlinear systems," IEEE Transactions on Systems, Man, and Cybernetics: Systems, vol. 47, no. 8, pp. 2209-2217, 2017.

[12] P. Balasubramaniam, P. Muthukumar, and K. Ratnavelu, "Theoretical and practical applications of fuzzy fractional integral sliding mode control for fractional-order dynamical system," Nonlinear Dynamics, vol. 80, no. 1-2, pp. 249-267, 2015.

[13] T. M. Duc, N. Van Hoa, and T.-P. Dao, "Adaptive fuzzy fractional-order nonsingular terminal sliding mode control for a class of second-order nonlinear systems," Journal of Computational and Nonlinear Dynamics, vol. 13, no. 3, p. 031004, 2018.

[14] S. Ha, H. Liu, S. Li, and A. Liu, "Backstepping-based adaptive fuzzy synchronization control for a class of fractional-order chaotic systems with input saturation," International Journal of Fuzzy Systems, vol. 21, no. 5, pp. 1571-1584, 2019.

[15] B. Zohoori, A. Verbraeck, M. Bagherpour, and M. Khakdaman, "Monitoring production time and cost performance by combining earned value analysis and adaptive fuzzy control," Computers \& Industrial Engineering, vol. 127, pp. 805-821, 2019.

[16] S. Masoudi, M. R. Soltanpour, and H. Abdollahi, "Adaptive fuzzy control method for a linear switched reluctance motor," IET Electric Power Applications, vol. 12, no. 9, pp. 1328-1336, 2018.

[17] H. F. Ghavidel and A. A. Kalat, "Observer-based hybrid adaptive fuzzy control for affine and nonaffine uncertain nonlinear systems," Neural Computing and Applications, vol. 30, no. 4, pp. 1187-1202, 2018. 
[18] Y. Zhou, H. Liu, J. Cao, and S. Li, "Composite learning fuzzy synchronization for incommensurate fractional-order chaotic systems with time-varying delays," International Journal of Adaptive Control and Signal Processing, vol. 33, no. 12, pp. 1739-1758, 2019.

[19] H. Liu, Y. Pan, J. Cao, Y. Zhou, and H. Wang, "Positivity and stability analysis for fractional-order delayed systems: a T-S fuzzy model approach," IEEE Transactions on Fuzzy Systems, p. 1, 2020.

[20] T. George Thuruthel, Y. Ansari, E. Falotico, and C. Laschi, "Control strategies for soft robotic manipulators: a survey," Soft Robotics, vol. 5, no. 2, pp. 149-163, 2018.

[21] Y. Pan, X. Li, and H. Yu, "Efficient pid tracking control of robotic manipulators driven by compliant actuators," IEEE Transactions on Control Systems Technology, vol. 27, no. 2, pp. 915-922, 2018.

[22] A. J. Muñoz-Vázquez, F. Gaxiola, F. Martínez-Reyes, and A. Manzo-Martínez, "A fuzzy fractional-order control of robotic manipulators with pid error manifolds," Applied Soft Computing, vol. 83, Article ID 105646, 2019.

[23] S. Yi and J. Zhai, "Adaptive second-order fast nonsingular terminal sliding mode control for robotic manipulators," ISA Transactions, vol. 90, pp. 41-51, 2019.

[24] W. He and Y. Dong, "Adaptive fuzzy neural network control for a constrained robot using impedance learning," IEEE Transactions on Neural Networks and Learning Systems, vol. 29, no. 4, pp. 1174-1186, 2018.

[25] Q. Zhou, H. Li, and P. Shi, "Decentralized adaptive fuzzy tracking control for robot finger dynamics," IEEE Transactions on Fuzzy Systems, vol. 23, no. 3, pp. 501-510, 2015.

[26] J. De Jesús Rubio, "Discrete time control based in neural networks for pendulums," Applied Soft Computing, vol. 68, pp. 821-832, 2018.

[27] J. De Jesús Rubio, E. Garcia, G. Aquino, C. Aguilar-Ibañez, J. Pacheco, and A. Zacarias, "Learning of operator hand movements via least angle regression to be teached in a manipulator," Evolving Systems, vol. 11, pp. 317-332, 2018.

[28] V. T. Yen, W. Y. Nan, and P. Van Cuong, "Recurrent fuzzy wavelet neural networks based on robust adaptive sliding mode control for industrial robot manipulators," Neural Computing and Applications, vol. 31, pp. 6945-6958, 2018.

[29] J. J. Rubio, "Modified optimal control with a backpropagation network for robotic arms," IET Control Theory \& Applications, vol. 6, no. 14, pp. 2216-2225, 2012. 